\title{
Prevalence of peripapillary choroidal neovascular membranes (PPCNV) in an elderly UK population-the Bridlington eye assessment project (BEAP): a cross-sectional study (2002-2006)
}

\author{
Craig Wilde ${ }^{1} \cdot$ Ali Poostchi $\mathbb{D}^{1} \cdot$ Rajnikant L. Mehta ${ }^{2} \cdot$ Jonathan G. Hillman ${ }^{3} \cdot$ Hamish K. MacNab $^{3} \cdot$ Marco Messina $^{1} \cdot$ \\ Gaspare Monaco $^{1} \cdot$ Stephen A. Vernon ${ }^{4} \cdot$ Winfried M. Amoaku $\mathbb{~ ( I ) ~}^{1}$
}

Received: 22 November 2017 / Revised: 1 February 2018 / Accepted: 5 June 2018 / Published online: 12 October 2018

(c) The Royal College of Ophthalmologists 2018

\begin{abstract}
Purpose There is paucity of data on the epidemiology of peripapillary choroidal neovascularisartion (PPCNV). Our aim was to determine prevalence of PPCNV in the elderly UK population of Bridlington residents aged $\geq 65$ years.

Methods Eyes with PPCNV in the Bridlington eye assessment project (BEAP) database of 3475 participants were analysed. PPCNV outline was drawn, its area measured, and clock-hour involvement of disc circumference recorded. Location and shortest distance from the lesion edge to fovea were recorded. Masked grading for age-related maculopathy (ARM)/reticular pseudodrusen (RPD) within the ETDRS grid was assigned for each eye using a modified Rotterdam scale. Peripapillary retinal pigment epithelial (RPE) changes/drusen were recorded. Visual acuity (VA) and demographic details analysed separately were merged with grading data.

Results PPCNV were identified in ten subjects, and were bilateral in two (20\%), a population prevalence of $0.29 \%$, and $0.06 \%$ bilaterality. Gender-specific prevalence were $0.36 \%$ and $0.19 \%$ for females and males, respectively. Age ranged from 66 to 85 years [mean 76.3 (SD 6.4)]. PPCNV were located nasal to disc in $41.7 \%$, measuring $0.46-7.93 \mathrm{~mm}^{2}$ [mean 2.81 $\mathrm{mm}^{2}$ (SD 2.82)]. All PPCNV eyes had peripapillary RPE changes. One subject had no ARM, 1 angioid streaks, and 30\% RPD. No direct foveal involvement, or reduced VA attributable to PPCNV was observed.

Conclusion PPCNV were infrequent in this population, more common in females, and often located nasal to the disc, without foveal extension. Peripapillary degenerative changes were universal, and strong association with ARM was observed in eyes with PPCNV. Typically, PPCNV were asymptomatic with VA preservation.
\end{abstract}

\section{Introduction}

Peripapillary choroidal neovascular membranes (PPCNV) form part of the spectrum of diseases that have potential to

Winfried M. Amoaku

wma@nottingham.ac.uk

1 Ophthalmology and Vision Sciences, Division of Clinical Neurosciences, B Floor, EENT Centre, Queen's Medical Centre, University of Nottingham, Nottingham, UK

2 Research Design Service, East Midlands (RDS EM), School of Medicine University of Nottingham, Nottingham Health Science Partners, QMC, Nottingham NG7 2UH, UK

3 The Medical Centre, Station Avenue, Bridlington YO16 4LZ, UK

4 University Hospital, Queen's Medical Centre, Nottingham and Honorary Professor of Ophthalmology, University of Nottingham, Nottingham, UK cause severe visual loss [1]. They are well-recognized but uncommon, reportedly accounting for less than $10 \%$ of all newly presenting choroidal neovascular membranes (CNV) $[2,3]$. In a survey by Browning and Fraser [4], PPCNVs were associated with age-related macular degeneration (AMD) in $45 \%$ of cases, while $39 \%$ were considered idiopathic. PPCNV are also associated with conditions such as inflammatory diseases including presumed ocular histoplasmosis [5], uveitis [6-9], and chorioretinitis [10], and degenerative processes including myopia [11] and angioid streaks [4]. Choroidal osteoma, optic disc drusen and congenital disc anomalies are other rare associations. Our group has previously reported that PPCNV occurred in 9 out of 231 cases of newly presenting CNV in a UK hospital setting (3.9\%) [2]. Previous reports on the prevalence and associations of PPCNV are based on case reports or small case series from hospital data. To the best of our knowledge, there are no reports on population prevalence of PPCNV 
except for our previous report of worse eye prevalence of $0.29 \%$ for Grade 4c (PPCNV), compared to $1.8 \%$ for neovascular AMD (nAMD) (grade 4b AMD) and $2.5 \%$ for geographic atrophy (GA) (grade 4a AMD) [12]. Furthermore, there are no data available in the literature on the population prevalence of asymptomatic PPCNV.

In this study, characteristics of eyes classified as having PPCNV from the Bridlington eye assessment project (BEAP) were investigated.

\section{Methods}

The BEAP study methods, including image acquisition and analysis, have been reported previously [12]. In summary, the BEAP is a single centre population-based prevalence study, with the primary objective to investigate the utility of screening for eye disease in an elderly population $\geq 65$ years, using clinical examination by trained optometrists and digital imaging technology. All individuals registered with a General Practitioner in Bridlington and 65 years and older on the 5th November 2002 were eligible for inclusion in the project. Subjects known to be moving in or out of the area during the study, those that were registered blind or partially sighted, bed bound individuals or those known to have significant dementia were excluded from the study. Subjects were invited by letter on a street-by-street basis in ascending numerical order of postcode. When contacted, each subject was invited to telephone the BEAP to make an appointment to be examined. At its completion in March 2006 over 3500 subjects had been examined. All participants were interviewed, in person, by a trained research nurse using structured questionnaire, and examined by one of four specially trained optometrists with structured proforma completed by the research staff. Non-stereoscopic mydriatic fundus photography was performed with a Topcon fundus camera (model TRC NW6S) and a Nikon 10-megapixel camera. Each eye had a $30^{\circ}$ colour fundus photograph (CFP) taken centred on the macula. Local ethics committee approval (Ref. no. PB/RH/02/288) was obtained and the research adhered to the tenets of the Declaration of Helsinki. All subjects provided informed consent. Masked image grading was performed in accordance with the International Classification System of AMD, using $30^{\circ}$ nonmydriatic colour fundus photographs. All photographs of right and left eyes were graded by a single ophthalmologist (CW) who was trained in image grading at the Central angiographic reading facility (CARF), Belfast, Northern Ireland. One in ten (1 in 10) randomly selected right eye images were sent to the CARF for secondary masked grading by certified graders.

The diagnosis of PPCNV was clinical, and based on surrogate clinical features of CNV (as previously described)
[12] directly adjacent to and contiguous with the optic disc. These include definite RPE detachment, haemorrhagic or serous, and/or subretinal or sub-RPE haemorrhages unassociated with any other vascular lesion and/or intraretinal, subretinal or sub-RPE glial tissue, and/or subretinal or subRPE neovascular membrane as characterized by grey/yellowish discoloration. All eyes identified as having PPCNV, or questionable lesions were reviewed and scrutinised by a retinal specialist (WMA). Any differences in opinion were sent to CARF for secondary grading. All images were analysed using the Topcon IMAGEnet 2000 programme. All eyes identified with PPCNV (and corresponding contralateral eyes) were subsequently reviewed in greater detail, with demographic details and visual acuity (VA) unknown to the grader. The outline of the PPCNV was drawn and area measured. Location of the lesion was also recorded. The PPCNV area was taken to include only visible membrane (as indicated by a grayish-brown or whitish-grey appearance) and/or areas of sub-retinal or subRPE haemorrhage. Areas of obvious peripapillary glial tissue, if associated with haemorrhage were also measured as part of the lesion. Often, the lesion would be located adjacent to the disc, with peripheral edge haemorrhage or exudate. Areas of subretinal fluid (SRF) without any haemorrhage were not in themselves measured as part of the lesion. Only areas of gross exudation immediately adjacent to haemorrhage or membrane were included in the measured area, whereas sparse distal exudates were not. The extent/severity of the PPCNV was recorded using clock hours of involvement of the optic disc circumference. An example of a PPCNV is shown in Fig. 1.

Signs of age-related maculopathy (ARM) within an ETDRS macular grid were recorded using a modified Rotterdam grade as previously described [12]. Retinal pigment epithelial (RPE) changes and presence of drusen in the

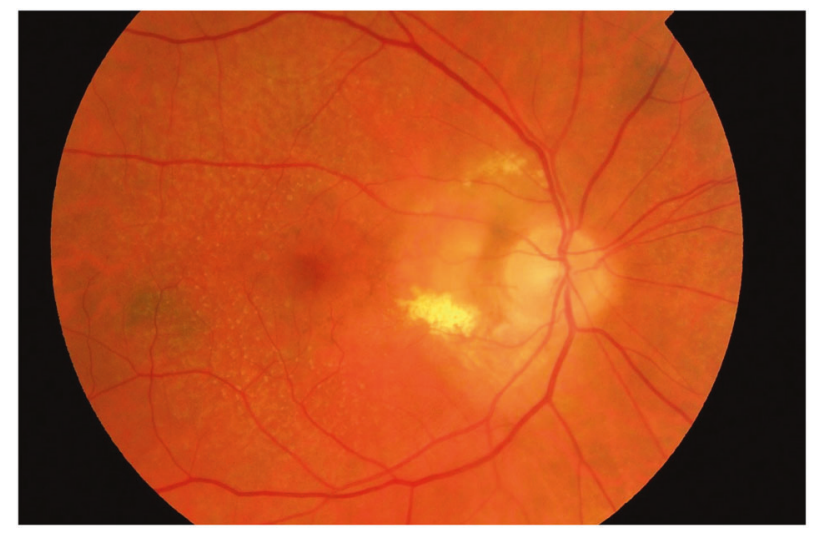

Fig. 1 Non-stereoscopic colour fundus photograph. A peripapillary choroidal neovascular membrane involving the temporal 6 clock hours of the optic disc circumference is present, and associated with exudation. There are visible co-morbid reticular pseudodrusen present in the macula and elsewhere 
Table 1 Populatio-based prevalence of PPCNV in the Bridlington eye assessment project (BEAP). Data is number (\%) [95\%CI]

\begin{tabular}{lllll}
\hline Grade & $\begin{array}{l}\text { Right eye (3340 gradable } \\
\text { photos) }\end{array}$ & $\begin{array}{l}\text { Left eye (3384 gradable } \\
\text { photos) }\end{array}$ & $\begin{array}{l}\text { Participants (3475 gradable } \\
\text { photos) }\end{array}$ & $\begin{array}{l}\text { Bilateral (3255 participants with } \\
\text { gradable photos in both) }\end{array}$ \\
\hline PPCNV/4c & $6(0.18)[0.07-0.40]$ & $6(0.18)[0.07-0.40]$ & $10(0.29)[0.15-0.54]$ & $2(0.06)[0.01-0.24]$ \\
$\begin{array}{l}\text { Mean age } \\
\text { (years) }\end{array}$ & 76.7 & 73.6 & 76.3 & 69.5 \\
\hline
\end{tabular}

Table 2 Age (years)-specific prevalence of PPCNV. Data is number $(\%)[95 \% \mathrm{CI}]$

\begin{tabular}{|c|c|c|c|c|c|c|c|}
\hline Age range (years) & $65-69$ & $70-74$ & $75-79$ & $80-84$ & $85-89$ & $\geq 90$ & Total \\
\hline $\begin{array}{l}\text { Number of PPCNV eyes } \\
(\%)[95 \% \mathrm{CI}]\end{array}$ & $\begin{array}{l}2(0.24)[0.01- \\
0.91]\end{array}$ & $\begin{array}{l}1(0.09)[0.01- \\
0.58]\end{array}$ & $\begin{array}{l}3(0.37)[0.07- \\
1.14]\end{array}$ & $\begin{array}{l}3(0.56)[0.11- \\
1.72]\end{array}$ & $\begin{array}{l}1(0.55)[0.01- \\
3.34]\end{array}$ & $\begin{array}{l}0(0.00)[0.00- \\
12.39]\end{array}$ & $\begin{array}{l}10(0.29) \\
{[0.15-0.54]}\end{array}$ \\
\hline $\begin{array}{l}\text { Total gradable eyes } \\
\text { available }\end{array}$ & 849 & 1069 & 808 & 533 & 183 & 33 & 3475 \\
\hline
\end{tabular}

Table 3 Summary of demographic details of eyes with PPCNV and associated macular age-related changes (Rotterdam Grades), with LogMAR visual acuity (VA)

\begin{tabular}{|c|c|c|c|c|c|c|c|c|}
\hline Number & Affected eye & $\begin{array}{l}\text { Age at } \\
\text { exam }\end{array}$ & Sex & $\begin{array}{l}\text { (Macular status) } \\
\text { AMD grade right }\end{array}$ & $\begin{array}{l}\text { (Macular status) } \\
\text { AMD grade left }\end{array}$ & $\begin{array}{l}\text { Right VA } \\
\text { (LogMAR) }\end{array}$ & $\begin{array}{l}\text { Left VA } \\
\text { (LogMAR) }\end{array}$ & $\begin{array}{l}\text { Cause of vision } \\
\text { loss }\end{array}$ \\
\hline 1 & LE & 66 & $\mathrm{~F}$ & $1 \mathrm{a}$ & $1 \mathrm{a}$ & 0.02 & 0.14 & Nil \\
\hline 2 & LE & 76 & $\mathrm{~F}$ & $1 \mathrm{a}$ & $1 \mathrm{a}$ & 0.30 & 0.42 & Other: cataracts \\
\hline 3 & LE & 76 & $\mathrm{~F}$ & $1 \mathrm{a}$ & $1 \mathrm{a}$ & 0.1 & 0.1 & Nil \\
\hline 4 & LE & 85 & M & $4 \mathrm{~b}$ & 4 , reticular drusen & $\mathrm{CF}$ & 0.3 & ARM \\
\hline 5 & $\mathrm{RE}$ & 83 & $\mathrm{~F}$ & $0 \mathrm{a}$ & $0 \mathrm{~b}$ & 0.2 & 0.2 & Nil \\
\hline 6 & $\mathrm{BE}$ & 72 & $\mathrm{~F}$ & $1 \mathrm{a}$ & $1 \mathrm{a}$ & 0.1 & 0.1 & Nil \\
\hline 7 & $\mathrm{RE}$ & 77 & M & $2 \mathrm{a}$, reticular drusen & $\begin{array}{l}\text { 2a, reticular } \\
\text { drusen }\end{array}$ & 0.2 & 0.2 & Nil \\
\hline 8 & $\mathrm{RE}$ & 80 & M & 3 , reticular drusen & 3 , reticular drusen & 0.0 & 0.0 & Nil \\
\hline 9 & $\mathrm{BE}$ & 67 & $\mathrm{~F}$ & $4 a$ & $4 \mathrm{a}$ & 0.3 & 0.4 & $\begin{array}{l}\text { RE AMD-GA, LE } \\
\text { diabetic CSMO }\end{array}$ \\
\hline $\begin{array}{l}10 \text { (angioid } \\
\text { streaks) }\end{array}$ & $\mathrm{RE}$ & 81 & $\mathrm{~F}$ & $1 \mathrm{a}$ & $\begin{array}{l}\text { Cannot grade } \\
\text { (Non-AMD) }\end{array}$ & 0.22 & 0.36 & $\begin{array}{l}\text { RE-ERM, LE- } \\
\text { cataract }\end{array}$ \\
\hline
\end{tabular}

peripapillary area (one-disc diameter around the optic disc) were specifically recorded along with the closest distance from the edge of changes secondary to the PPCNV, including SRF to the fovea. ARM changes in the contralateral eyes were recorded.

VA and demographic details which were analysed separately were then merged with the image grading data.

Statistical analysis was performed using Stata 12.0 (StataCorp, College Station, TX) and SPSS v.22 (IBM Corp. Armonk, NY). Patient demographic characteristics that are categorical will be summarised using percentages and continuous normally distributed variables with the presentation of means, standard deviation and associated $95 \%$ confidence intervals.

Where the continuous variable is non-normally distributed we will present medians and associated quartiles (25th, 75th). Statistical significance will be shown when $P$-values $<0.05$.

\section{Results}

Amongst the total of 3475 participants with gradable photographs in at least one eye, PPCNV were identified in ten subjects (Table 1), with bilateral occurrence in two individuals $(20 \%)$. This resulted in a total of 12 eyes with PPCNV, and an overall population prevalence of $0.29 \%$. PPCNV accounted for $12(13.3 \%)$ of a total of 90 cases of CNVMs identified in the BEAP Study. Seven individuals (70\%) were female resulting in gender-specific prevalence rates of $0.36 \%(7 / 1939)$ and $0.19 \%(3 / 1536)$ for females and males respectively. Bilateral involvement equated to a population prevalence of $0.06 \%$. Ages ranged from 66 to 85 years, with a mean of 76.3 years (SD 6.4). There was no obvious increase in prevalence with age, as shown in Table $2(p=0.77)$.

Eyes with PPCNV had a higher prevalence of the more advanced stages of AMD when compared to the overall 


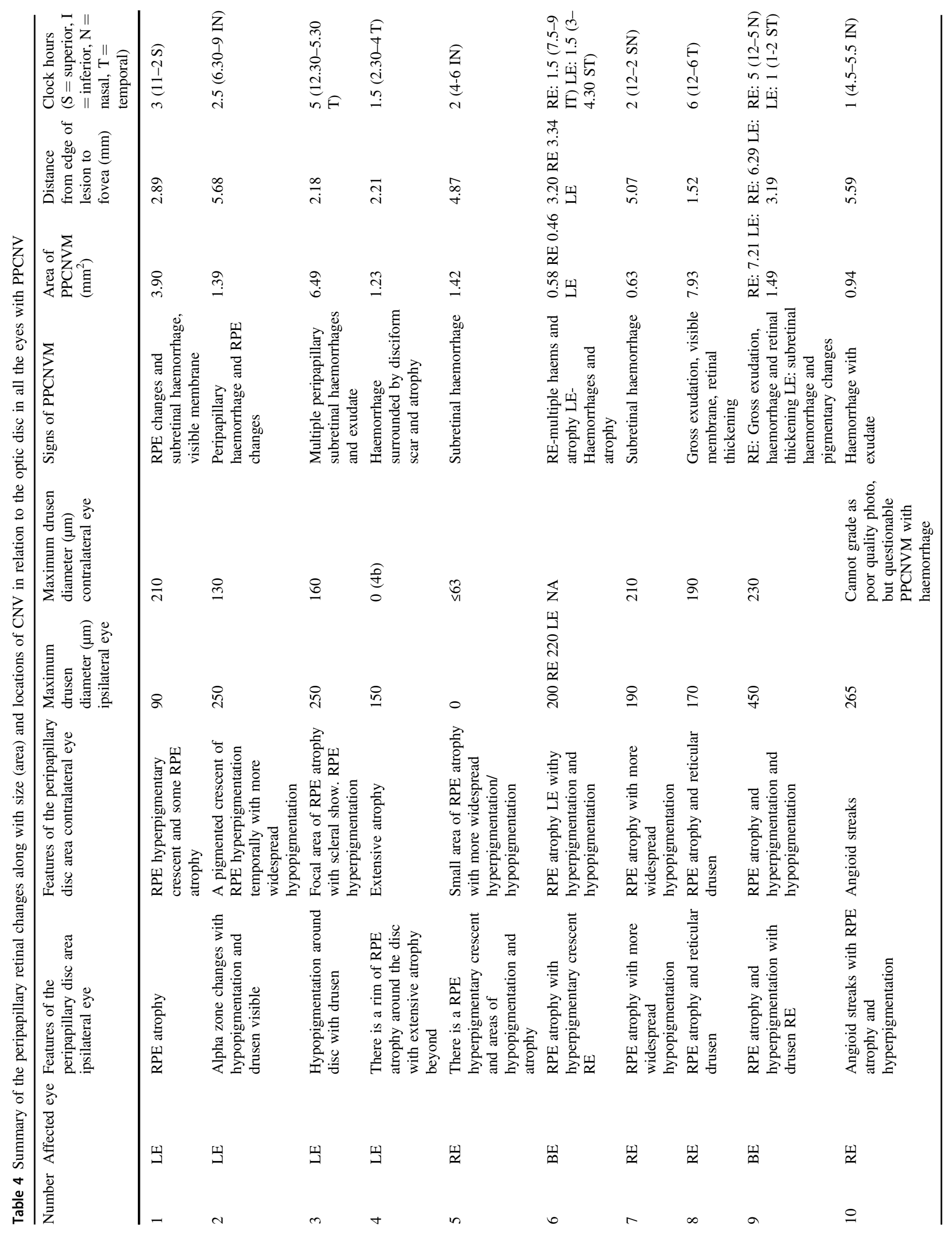


BEAP cohort. The prevalence of Rotterdam grade 2, 3 or $4 \mathrm{a}$ within the macula in PPCNV eyes was considerably high at $42 \%$ compared to that in the general population (see Tables 3 and 4). Only 1 eye in the ten individuals with a PPCNV had minimal/no sign of ARM present (Rotterdam grade 0$)$.

One subject $(10 \%)$ had identifiable angioid streaks. This individual was removed from our initial report of AMD prevalence [12] as the pathology was not felt to be purely age-related. The patient was 81 years of age and had comorbid large soft drusen within the macula. No individuals with PPCNV had pathological myopia or optic disc pathology, such as disc drusen or disc swelling, or evidence of previous chorioretinitis. None of the eyes with PPCNV had direct involvement of their fovea with SRF, exudate or haemorrhage in the photographs. Visual function was good in all eyes, with none having poor VA secondary to PPCNV. Eyes with reduced vision had co-morbid conditions as summarised in Table 3. Patient number 10 (LogMAR VA 0.22, Snellen VA=20/32) had a co-morbid epiretinal membrane. Patient 2 (LogMAR VA of 0.42 [Snellen 20/50]), subsequently underwent cataract surgery and postoperatively had a VA of 0.2 [Snellen 20/30]. Subject 4 had a LogMAR VA of 0.3 [Snellen 20/40] at presentation. This eye had a 23-months follow-up in a hospital eye service, after which the patient was reported to have a haemorrhagic peripapillary scar with atrophy and discharged from further follow-up. The VA reduction was attributed in part to secondary macula RPE atrophic changes.

Table 3 details demographic and macular changes in participants with PPCVN. Table 4 summarises the peripapillary retinal changes, along with size (area) and locations of CNV in relation to the optic disc in all eyes with PPCNV. Three eyes of 3 subjects $(30 \%)$ with PPCNV had reticular pseudodrusen (RPD). Nine out of ten individuals (90\%) had evidence of drusen $\geq 63 \mu \mathrm{m}$ in size within the macular area, including the one with angioid streaks. One individual (10\%) had contralateral nAMD; another had bilateral GA with multiple large drusenoid pigment epithelial detachments that appeared completely separate from any PPCNV (which was nasal to the disc). All eyes with PPCNV had RPE hyperpigmentary, hypopigmentary or atrophic changes around the optic disc. One eye $(8.33 \%)$ had a large PPCNV involving $\geq 6$ clock hours. Six of the 12 identified PPCNM had predominantly temporal location with another occurring superiorly. Five of 12 PPCNVs (41.7\%) involved retina nasal to the optic disc only. The PPCNV size ranged from $0.46 \mathrm{~mm}^{2}$ to $7.93 \mathrm{~mm}^{2}$ with a mean of $2.81 \mathrm{~mm}^{2}$ (SD 2.82, 95\% CI 1.1-4.6). The mean area of PPCNV in the four eyes with visible exudation was $5.64 \mathrm{~mm}^{2}$ (95\% CI 0.57-10.72). For the eight eyes with no visible exudation, the membranes measured an average of $1.39 \mathrm{~mm}^{2}$ (95\% CI $\left.0.47-2.31\right)$.

\section{Discussion}

This study reports the prevalence of PPCNV within an elderly Caucasian population, using data from the largest UK population based screening study of AMD to date. To the best of our knowledge, this represents the first population study specifically to report on the prevalence of PPCNV, as well as describe the characteristics of PPCNV and concomitant ocular findings in affected and fellow eyes, as all previous publications on the subject were based on hospital cohorts, in predominantly symptomatic individuals, with inherent selection bias. Our results indicate that as many as two thirds of PPCNV may remain asymptomatic, compatible with the finding that $40 \%$ of the lesions are located nasal to the disc.

PPCNVs (grade 4c AMD) were an infrequent finding, considerably lower than the prevalence of grade 4b AMD, in the same population [12]. Our findings are similar to those of some previous published studies [13, 14], where there was a female preponderance. RPD are known to have such female predilection $[12,15]$. However, it is unlikely that the increased female prevalence of PPCNV in this study is due to the presence of RPD, as the three patients with RPD and PPCNV in the series were male. We have previously reported that PPCNV accounted for $3.9 \%$ of newly diagnosed cases of CNV in AMD in a hospital eye service [2]. Other investigators have reported frequency of $<10 \%$ of all CNVs [16]. This relatively low prevalence is confirmed in this study, where PPCNV accounted for $13.3 \%$ of all identified CNVs.

The majority of PPCNVs in this cross-sectional study were unilateral, similar to what was reported at baseline in hospital based longitudinal studies [4, 14]. In the Silvestri study [14], only two out of the 14 (14\%) individuals had bilateral PPCNV at their initial presentation, whilst others developed contralateral eye involvement over follow up of up to 7 years resulting in 54\% bilteralism [14]. Similarly, Browning et al. reported bilateral involvement in 19 of their 96 patients (19.8\%) [4] after a median follow up of 2 years.

There is a myriad of reported associations between PPCNV and other conditions, most of which are based on single case reports or small case series. Larger hospitalbased studies also exist, but may give a poor representation of the true spectrum of disease as small, nasal, age-related PPCNVs may remain asymptomatic. In a series of 115 eyes of 96 patients, Browning et al. reported ocular conditions associated with PPCNV as 45.2\% ARM, 39.1\% idiopathic, 4.3\% multifocal choroiditis, $2.6 \%$ angioid streaks, $1.7 \%$ presumed ocular histoplasmosis, $1.7 \%$ choroidal osteoma, $0.9 \%$ optic disc drusen and $0.9 \%$ congenital disc anomalies [4]. The definition of ARM was however broad, and included all eyes with $\geq 1$ of drusen $>63 \mu \mathrm{m}$, pigment clumps, mottled pigment epithelial atrophy, GA and signs 
of an exudative AMD, including disciform scars in both eyes [4]. They also reported that $39 \%$ of subjects with PPCNV had drusen on colour photography [4]. Kies and Bird similarly reported 15 of their 55 eyes (27\%) with PPCNV had identifiable drusen [13], and Silvestri et al. reported that $60 \%$ of PPCNV were related to age-related degenerative changes [14]. In the present series, the presence of drusen $\geq 63 \mu \mathrm{m}$ within the macular area in $90 \%$ of cases was much higher than previously published. This higher prevalence of PPCNV in eyes with the more advanced stages of AMD when compared to the overall BEAP cohort could be a chance finding, but suggests a stronger association of PPCNV with ARM than previously published. The lower prevalence of ARM reported within hospital populations could, in part, be explained by the fact that these are symptomatic, and more likely to be large lesions with macula involvement from exudation or SRF which could mask drusen or result in their regression. Alternatively, it could reflect the older age of the cohort included in the present study. RPD or subretinal drusenoid deposits (SDD) cover a large area of the retina including the peripapillary zone $[15,17]$, and are known to be associated with nAMD [18-20]. We have previously reported their prevalence in $22 \%$ of eyes with newly presenting nAMD in a hospital eye service [19], a finding similar to that in participants with PPCNV in this population.

Peripapillary degenerative changes in the present series were a more universal finding when compared to the presence of macular drusen. Previous studies on PPCNV have not reported the presence of drusen or pigmentary change in the immediate peripapillary area. Recent studies have reported the presence of peripapillary SDD in association with drusen and other age-related changes using multimodal imaging $[15,17]$. As drusen and pigmentary changes within the macula are known hallmarks of both GA and CNV, and SDD predispose to CNV, it seems logical to consider these changes in the peripapillary area as potentially pathological for PPCNV. Older reports have speculated on potential relationship of previous unwitnessed episodes of multiple white dot syndromes in the aetiology of the relatively large cohort of presumed idiopathic PPCNVs [21, 22]. A more plausible explanation would be that localized, age-related changes occurring in the peripapillary area may predispose to localized breaks in Bruch's membrane allowing CNV membranes to develop as reported by Sarks [23].

The association with angioid streaks and PPCNV is well established [24-27]. The number of such cases is small, but suggests that membranes occurring in the region of angioid streaks remain small and asymptomatic and, therefore, are less likely to present to a hospital eye service. Potential overlaps between the aetiologies of PPCNV in patients with angioid streaks and comorbid age-related changes are possible.
Some authors have defined a PPCNV as large if it covered more than 3.5 disc areas or involved over $50 \%$ of the disc circumference [28]. Only one subject $(10 \%)$ in the current series had $>6$ clock hours involved. This figure is similar to the $15 \%$ prevalence of large membranes reported by Browning et al. [4], but less than the higher prevalence of $87 \%$ of large membranes involving more than 6 clock hours reported by Kies and Bird [13]. There was complete disc encirclement by the PPCNV in $0.9 \%$ of Browning's cases [4] whilst $11 \%$ in Kies and Bird series [13] had similar features [4], probably reflecting selection bias. Caution should however be exercised when comparing the size of CNV lesions, given the different imaging modalities used in the different studies, especially as PPCNV may be larger in surgical excision and indocyanine green angiography than on FFA [13, 28-30].

The finding of asymptomatic PPCNVs in this study is interesting. Our literature search could not find any studies on the prevalence of asymptomatic PPCNV. Sarks provides the best insight on the subject, with a clinicopathological correlative study of 150 eyes of 80 patients obtained postmortem [23], which identified CNVMs in the peripapillary area in $14 \%$, macular area in $20 \%$, and in the peripheral retina in $24.6 \%$ of eyes. Unlike the large temporal PPCNVs seen in eyes of symptomatic individuals which had extended to the fovea [14, 23, 31], Sarks suggested that in older subjects, small and frequently nasal asymptomatic PPCNV occurred with greater frequency than large, temporal membranes with macula involvement, and demonstrated that PPCNVs originate from choroidal vessels passing either through breaks in Bruch's membrane (57\%) or from vessels extending around the termination of Bruch's membrane $(43 \%)$ especially on the nasal side [23].

Limitations of our study include utilization only of CFP of field 2. Although the disc and nasal peripapillary areas were well included in these images, the addition of CFP of field 1, centered on the optic disc may have added clearer delineation of nasal PPCNV. Multimodal imaging including SD-OCT around the optic disc, combined with angiography in suspected cases would also have improved case detection, and are recommended in future population studies. Individuals registered as blind or partially sighted were excluded from study inclusion. However, our analysis showed that such subjects were few, and had subfoveal $\mathrm{CNV}$. We cannot be sure that some of these individuals may have had poor vision secondary to PPCNV which could be bilateral or in combination with other visually significant ocular pathology. Such scenarios would make our prevalence measure an underestimation.

In conclusion, this study confirms that PPCNV are an infrequent finding in the elderly population compared to macular $\mathrm{CNV}$, and have a clear female preponderance. PPCNV may be asymptomatic especially when small or 
located nasally. This finding is very distinct from that in previous hospital studies of symptomatic patients, and supports the early histopathological study [23] which suggested that in elderly individuals, PPCNV frequently occurred in a nasal position and are asymptomatic in the majority of cases. This study also reports the universal finding of peripapillary degenerative changes in all eyes with PPCNV, and a strong association between PPCNV and signs of ARM within both the macula and immediate peripapillary areas plus association with RPD. Multimodal imaging such as OCT, FFA and ICG would help identify possible phenotypic variants. More research is also required on the natural history of these lesions, including those that are located nasally.

\section{Summary}

\section{What was known before}

- There are some data on the occurrence and natural history of peripapillary choroidal neovascularisation (PPCNV) in hospital settings but a paucity of data exists on the epidemiology of PPCNV in community populations.

- There is no detailed description of the prevalence and features of asymptomatic PPCNV.

\section{What this study adds}

- This represents the first study to report PPCNV occurs with a population prevalence of $0.29 \%$, and is bilateral in $0.06 \%$ in the UK Caucasian population $\geq 65$ years.

- Gender-specific prevalence was $0.36 \%$ and $0.19 \%$ for females and males, respectively.

- PPCNV are commonly located nasal to the optic disc and are small and asymptomatic early on.

- Peripapillary RPE degenerative changes, drusen and RPD, as well as higher grade ARM predispose to PPCNV.

Acknowledgements This research was funded in part by a Research Grant from the Macular Society UK, Andover, Hants, UK. The Bridlington Eye Assessment Project was funded by an unrestricted grant from Pfizer. We would also like to thank the following organisations for financial support of the Project: Pharmacia, Yorkshire Wolds and Coast Primary Care Trust, The Lords Feoffees of Bridlington, Bridlington Hospital League of Friends, The Hull and East Riding Charitable Trust, The National Eye Research Centre (Yorkshire), The Rotary Club of Bridlington, The Alexander Pigott Wernher Memorial Trust, Bridlington Lions Club, The Inner Wheel Club of Bridlington, Soroptimist International of Bridlington, and The Patricia and Donald Shepherd Charitable Trust. The authors thank Sheila MacNab (Project Manager), and Stephen Brown, Janet Button, Graham Langton, and
Mark Kunz (Optometrists) for their work with the Project; John Bapty, Nigel Connell, Peter Jay, and Gillian Poole for their work as the charity trustees of the Bridlington Eye Assessment Project.

Author contributions WMA: consultant for Abbvie, Alimera, Allergan Inc, Bayer, Novartis, Pfizer, Santen, and Thrombogenics, and has undertaken research sponsored by Allergan, Novartis, and Pfizer. He has received speaker fees and travel grants from Allergan, Bausch and Lomb, Bayer, Novartis and Pfizer. JGH: none. HKM: none. RM: none. MM: none. GM: none. AP: none. SAV: consultant for Allergan and Alcon, and has undertaken research sponsored by Allergan and Pfizer. He has received speaker fees from Allergan, Alcon, Pfizer, Optivue and Topcon. CW: none.

\section{Compliance with ethical standards}

Conflict of interest The authors declare that they have no conflict of interest.

\section{References}

1. Lopez PF, Green WR. Peripapillary subretinal neovascularization. A review. Retina. 1992;12:147-71.

2. Wilde C, Patel M, Lakshmanan A, Amankwah R, Dhar-Munshi S, Amoaku W. The diagnostic accuracy of spectral-domain optical coherence tomography for neovascular age-related macular degeneration: a comparison with fundus fluorescein angiography. Eye. 2015;29:602-9.

3. Ruben S, Palmer H, Marsh RJ. The visual outcome of peripapillary choroidal neovascular membranes. Acta Ophthalmol. 1994;72:118-21.

4. Browning DJ, Fraser CM. Ocular conditions associated with peripapillary subretinal neovascularization, their relative frequencies, and associated outcomes. Ophthalmology. 2005; 112:1054-61.

5. Cantrill HL, Burgess D. Peripapillary neovascular membranes in presumed ocular histoplasmosis. Am J Ophthalmol. 1980;89:192203.

6. Arkfeld DF, Brockhurst RJ. Peripapillary subretinal neovascularization in peripheral uveitis. Retina. 1985;5:157-60.

7. Garcia CA, Segundo Pde S, Garcia Filho CA, et al. Intermediate uveitis complicated by choroidal granuloma following subretinal neovascular membrane: case reports. Arq Bras Oftalmol. 2008;71:890-3.

8. Mehta S, Hariharan L, Ho AC, et al. Peripapillary choroidal neovascularization in pars planitis. J Ophthalmic Inflamm Infect. 2013;3:13.

9. Shoughy SS, Jaroudi MO, Tabbara KF. Regression of peripapillary choroidal neovascular membrane in a patient with sarcoidosis after oral steroid therapy. Saudi J Ophthalmol. 2014;28:160-2.

10. Jampol LM, Orth D, Daily MJ, et al. Subretinal neovascularization with geographic (serpiginous) choroiditis. Am J Ophthalmol. 1979;88:683-9.

11. Hotchkiss ML, Fine SL. Pathologic myopia and choroidal neovascularization. Am J Ophthalmol. 1981;91:177-83.

12. Wilde C, Poostchi A, Mehta RL, MacNab HK, Hillman JG, Vernon SA, et al. Prevalence of age-related macular degeneration in an elderly UK Caucasian population-The Bridlington Eye Assessment Project: a cross-sectional study. Eye. 2017;31:104250. https://doi.org/10.1038/eye.2017.30

13. Kies JC, Bird AC. Juxtapapillary choroidal neovascularization in older patients. Am J Ophthalmol. 1988;105:11-9. 
14. Silvestri G, Archer DB, Johnston PB. Peripapillary subretinal neovascular membranes: the natural history. Eye. 1993; 7:398-402.

15. Zarubina AV, Neely DC, Clark ME, Huisingh CE, Samuels BC, Zhang Y, et al. Prevalence of subretinal drusenoid deposits in Older Persons with and without age-related macular degeneration by multimodal imaging. Ophthalmology. 2016;123: $1090-1100$

16. Berkow JW. Subretinal neovascularization in senile macular degeneration. Am J Ophthalmol. 1984;97:143-7.

17. Huisingh C, McGwin G Jr, Neely D, Zarubina A, Clark M, Zhang $\mathrm{Y}$, et al. The association between subretinal drusenoid deposits in older adults in normal macular health and incident age-related macular degeneration. Invest Ophthalmol Vis Sci. 2016; 57:739-45.

18. Cohen SY, m Dubois L, Tadayani R, Delahaye-Mazza C, Debibie C, Quentel G. Prevalence of reticular pseudodrusen in age-related macular degeneration with newly diagnosed choroidal neovascularisation. Br J Ophthalmol. 2007;91:354-9.

19. Wilde C, Patel M, Lakshmanan A, Morales MA, Dhar-Munshi S, Amoaku WM. Prevalence of reticular pseudodrusen in eyes with newly presenting neovascular age-related macular degeneration. Eur J Ophthalmol. 2015;26:128-34.

20. Hogg RE, Silva R, Staurenghi G, Murphy G, Santos AR, Rosina $\mathrm{C}$, et al. Clinical characteristics of reticular pseudodrusen in the fellow eye of patients with unilateral neovascular age-related macular degeneration. Ophthalmology. 2014;121:1748-55.
21. Oh KT, Christmas NJ, Russell SR. Late recurrence and choroidal neovascularization in multiple evanescent white dot syndrome. Retina. 2001;21:182-4.

22. Wyhinny GJ, Jackson JL, Jampol LM, Caro NC. Subretinal neovascularization following multiple evanescent white-dot syndrome. Arch Ophthalmol. 1990;108:1384-5.

23. Sarks SH. New vessel formation beneath the retinal pigment epithelium in senile eyes. Br J Ophthalmol. 1973;57:951-65.

24. Ballatori N, Clarkson TW. Developmental changes in the biliary excretion of methylmercury and glutathione. Science. 1982;216:61-3.

25. Singerman LJ, Hatem G. Laser treatment of choroidal neovascular membranes in angioid streaks. Retina. 1981;1:75-83.

26. Mansour AM, Shields JA, Annesley WH Jr., et al. Macular degeneration in angioid streaks. Int J Ophthal. 1988;197:36-41.

27. Lim JI, Bressler NM, Marsh MJ, Bressler SB. Laser treatment of choroidal neovascularization in patients with angioid streaks. Am J Ophthal. 1993;116:414-23.

28. Binder S. Surgical treatment of peripapillary choroidal neovascularisation. Br J Ophthalmol. 2007;91:990-1.

29. Kokame GT, Yamaoka S. Subretinal surgery for peripapillary subretinal neovascular membranes. Retina. 2005;25:564-9.

30. Wolf S, Wald KJ, Remky A, Arend O, Reim M. Evolving peripapillary choroidal neovascular membrane demonstrated by indocyanine green choroidal angiography. Retina. 1994;14:465-7.

31. Gass JD. Drusen and disciform macular detachment and degeneration. Arch Ophthalmol. 1973;90:206-17. 